\title{
Comparison of two Metal Ion Implantation Techniques for Fabrication of Gold and Titanium Based Compliant Electrodes on Polydimethylsiloxane
}

Muhamed Niklaus, Samuel Rosset, Philippe Dubois, Herbert R. Shea Ecole Polytechnique Fédérale de Lausanne (EPFL), Institute of Microengineering (IMT), Microsystems for Space Technologies Laboratory (LMTS), Rue Jaquet-Droz 1, 2002 Neuchâtel, Switzerland.

\section{ABSTRACT}

This study contrasts the implantation of $25 \mu \mathrm{m}$ thick Polydimethylsiloxane (PDMS) membranes with titanium and gold ions at $10 \mathrm{keV}$ and $35 \mathrm{keV}$ for doses from $1 \times 10^{15} \mathrm{at} / \mathrm{cm}^{2}$ to $2.5 \times 10^{16} \mathrm{at} / \mathrm{cm}^{2}$ implanted with two different techniques: Filtered Cathodic Vacuum Arc (FCVA) and Low Energy Broad Ion Beam (LEI). The influence of the ion energy, ion type, and implantation tool on the Young's modulus (E), resistivity and structural properties (nanocluster size and location, surface roughness) of PDMS membranes is reported. At a dose of $2.5 \times 10^{16}$ $\mathrm{at} / \mathrm{cm}^{2}$ and an energy of $10 \mathrm{keV}$, which for FCVA yields sheet resistance of less than 200 $\Omega$ /square, the initial value of $\mathrm{E}(0.85 \mathrm{MPa})$ increases much less for FCVA than for LEI. For gold we obtain $\mathrm{E}$ of $5 \mathrm{MPa}$ (FCAV) compared to $86 \mathrm{MPa}$ (LEI) and for titanium $0.94 \mathrm{MPa}$ (FCVA) compared to $57 \mathrm{MPa}$ (LEI). Resistivity measurements show better durability for LEI than for FCVA implanted samples and better time stability for gold than for titanium.

\section{INTRODUCTION}

Using metal ion implantation (MII) we developed a method that allows micropatterning of very compliant and optically transparent electrodes that can sustain high strains and millions of cycles, and can be used to fabricate buckling type membrane actuators with a vertical displacement of more than $25 \%$ of the membrane's diameter [1]. Starting with a PDMS membrane with a Young's modulus $0.85 \mathrm{MPa}, \mathrm{Au}$ ion implantation at $5 \mathrm{keV}$ for doses of order $10^{16}$ ions $/ \mathrm{cm}^{2}$ results in less than $1 \mathrm{MPa}$ increase in Young's modulus, electrical conductivity below $1 \mathrm{k} \Omega /$ square, and strain of over $175 \%$ can be reached while remaining conductive.

In this paper we will present the influence of two different implantation techniques on mechanical, structural and electrical properties of Polydimethylsiloxane (PDMS) implanted with $\mathrm{Ti}$ and $\mathrm{Au}$, at $10 \mathrm{keV}$ and $35 \mathrm{keV}$, and doses between $0.1 \times 10^{16} \mathrm{at} / \mathrm{cm}^{2}$ and $2.5 \times 10^{16} \mathrm{at} / \mathrm{cm}^{2}$.

\section{EXPERIMENTAL SETUP}

The two implantation instruments, used in this study, were Filtered Cathode Vacuum Arc (FCVA) and Low Energy Broad Beam (LEI) implanter. FCVA creates $600 \mu$ s long pulses of plasma with a beam current of $300 \mu \mathrm{A} / \mathrm{cm}^{2}$. Pulse rate is of order $1 \mathrm{~Hz}$. The beam is filtered from macroparticles, yielding mostly doubly charged ions. In our case, the beam is then accelerated toward the target with an acceleration potential of $5 \mathrm{kV}$. The acceleration potential drops during each pulse because of the current from the $17.5 \times 10^{12} \mathrm{at} / \mathrm{cm}^{2}$ doubly charged ions. LEI delivers a continuous ion current of about $0.5 \mu \mathrm{A} / \mathrm{cm}^{2}$. The ion beam is filtered, accelerated and decelerated 
at the desired energy. Beam energy is stable in time. Averaged over one second, the ion currents are in the same range for both implanters, but during a pulse, the ion current produced by FCVA is much higher than that produced by LEI.

Resistance measurements were performed on $25 \mu \mathrm{m}$ thick PDMS on Si substrates. Data for surface roughness, dose, mechanical and structural properties were obtained on $25 \mu \mathrm{m}$ thick PDMS films bonded on patterned silicon chips with $2 \mathrm{~mm}$ and $3 \mathrm{~mm}$ through-holes. The initial Young's modulus was $0.85 \mathrm{MPa}$. The surface roughness was measured with a Digital Instruments D3100 AFM. The TEM images were obtained with TEM Philips CM, and TEM samples were prepared using a Cryo-ultra-microtome (Leica Ultracut E) at $-130^{\circ} \mathrm{C}$ as described in detail in [2]. A bulge Test setup was used to extract the elasticity of membranes. Once implanted the doses were determined with Rutherford Backscattering Spectroscopy (RBS).

\section{RESULTS}

MII creates a nano-composite a few nm thick, located in the top tens of nm of the surface of the elastomer. The particle density and size distribution of clusters depend on the dose, as well as on diffusion. The depth of the implanted layer depends on the ion energy. The thickness of the layers varies according to the ion type and implantation condition, and can be approximately computed for low doses with the TRIM simulation program [3]. Figure 1 shows higher implantation energies lead to larger ion distribution and deeper ion penetration. Gold ions do not penetrate as deeply as titanium, because the stopping cross-section of the ion is proportional to its mass. Hence one may expect gold to form a metallic conducting network at a lower dose and energy than titanium.

\section{$\underline{\text { Resistance }}$}

An important increase of electrical conduction is one of the consequences resulting from MII. The conductivity rises with the dose and depends additionally on ion energy and intrinsic properties of the implanted element (e.g. electronegativity). Microscopically the phenomenon of conduction relates to the size and concentration of the clusters, as well as distance in-between (see figures 3 and 4 for microstructure). As the dose increases, inter-particle spacing decreases as they're getting bigger, till the point where first randomly connected conduction path is formed. This domain is characterized by an abrupt decrease of polymer's resistivity and is referred to as percolation domain. Above this dose the continuous metallic layer is smoothly formed and electrical properties vary only moderately with the concentration, approaching slowly to those of a thin metallic film. The electrical properties of the implanted PDMS are presented in figure 2 and are very well described by the percolation model.

The highest ratio of the electrical conductivity to dose is obtained for gold ions implanted with FCVA at $10 \mathrm{keV}\left(300 \Omega / \mathrm{square}\right.$ at $\left.1.75 \times 10^{16} \mathrm{at} / \mathrm{cm}^{2}\right)$. Gold is a better conductor than titanium and also FCVA produces a narrower distribution of ions due to the drop of the acceleration potential: during each pulse, the gold is initially implanted, and the finally deposited as the acceleration voltage drops (see TEM cross-sections in figure 3 and 4). The slowest decrease of resistance with the dose was measured on the $35 \mathrm{keV}$ titanium samples, as expected from the TRIM simulation. 

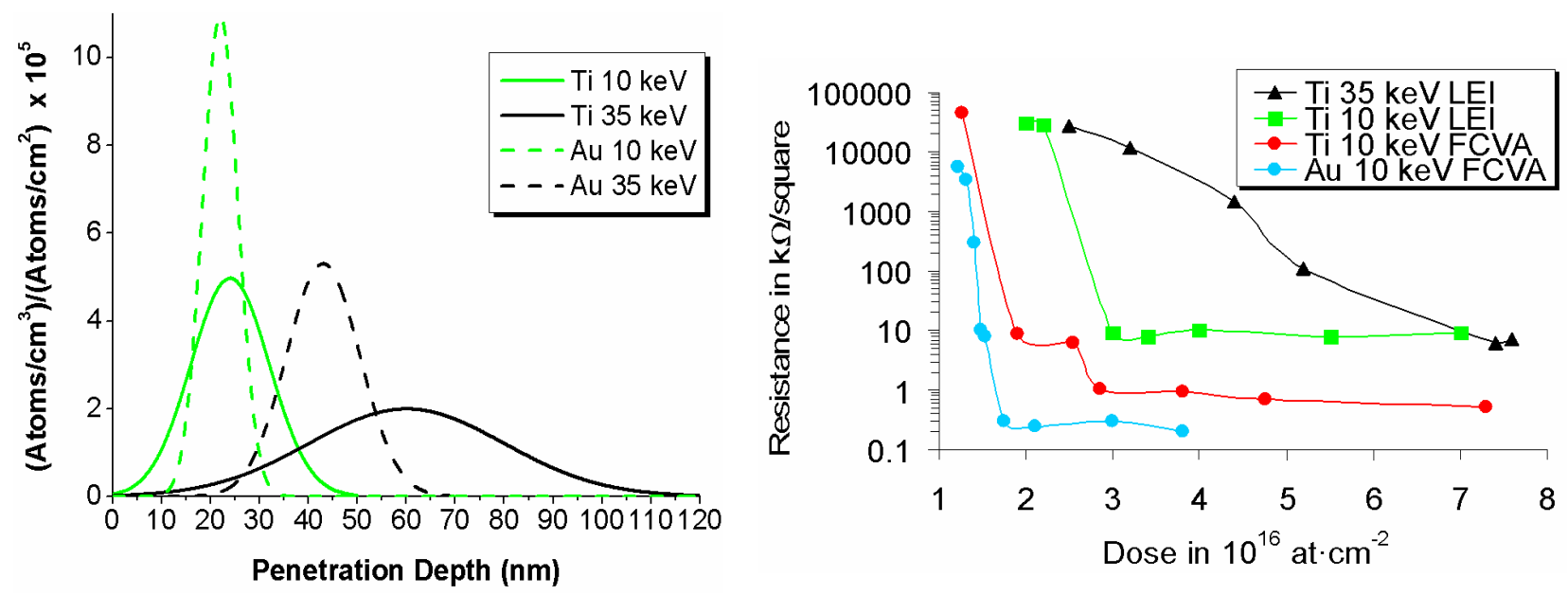

Figure 1. TRIM simulation of $\mathrm{Ti}$ and $\mathrm{Au}$ ion penetration depth into PDMS versus ion energy. Lower energy leads to denser distribution of ions closer to the surface.

Figure 2. Surface resistance vs. measured ion dose for different energies and implantation techniques. No data is shown for gold implanted by LEI since sputtering prevents obtained conductive layers.

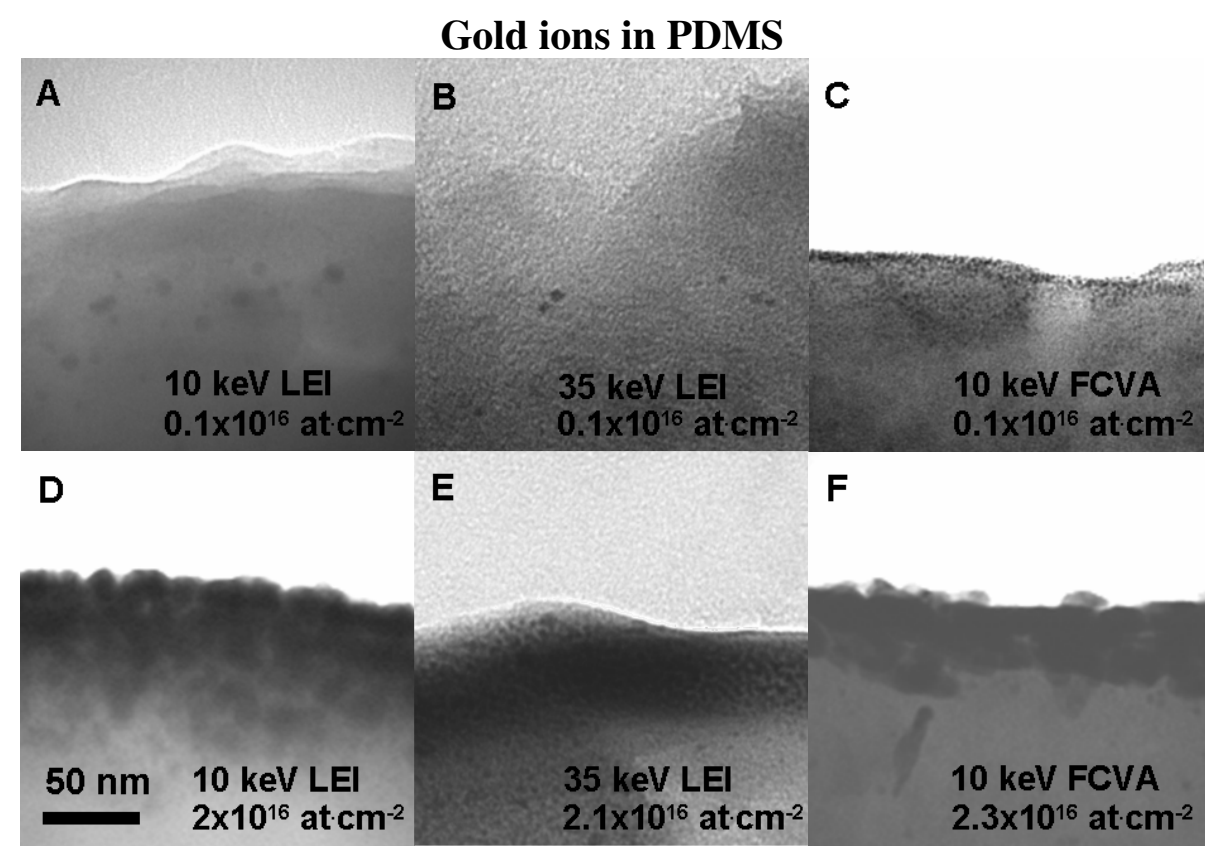

Figure 3. TEM micrographs and cluster morphology of PDMS following Gold ion implantation at 10 and $35 \mathrm{keV}$ using LEI and FCVA. Au implantation leads to round crystalline clusters of diameter up to $30 \mathrm{~nm}$. Doses are measured by RBS. The scale bar is for all 6 photographs. The PDMS is the gray area on the bottom half of each frame. The gold particles are darker, and the carbon grid is white or light gray. The particles depth ranges from $0 \mathrm{~nm}$ to roughly $40 \mathrm{~nm}$.

No resistance could be measured for the gold samples implanted with LEI, which is explained by the limited ion concentration. The maximal dose measured by RBS for LEI samples was $2.4 \times 10^{16} \mathrm{at} / \mathrm{cm}^{2}$. The reason for this is the sputtering of the composite that leads to a steady state condition of the ion concentration. 
Titanium ions in PDMS

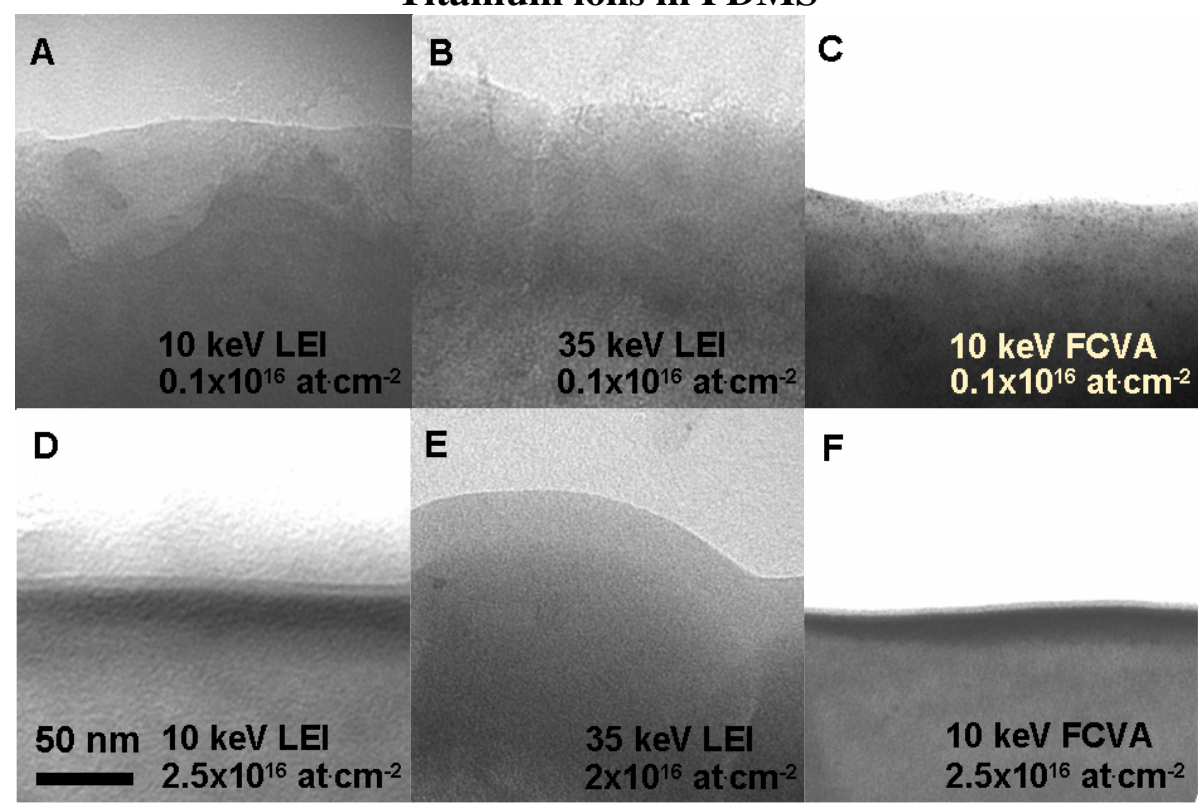

Figure 4. TEM micrographs and cluster morphology of PDMS following Titanium ion implantation at 10 and $35 \mathrm{keV}$ using LEI and FCVA. Ti implantation leads to nm-size amorphous aggregates unlike gold nanoparticles in Figure 3.

Time stability measurements of the conductivity showed that for FCVA implanted samples only the gold samples with doses above $1.75 \times 10^{16} \mathrm{at} / \mathrm{cm}^{2}$ retained the small electrical resistance. For the higher does, the conductivity remains constant for more than 2 years. At lower doses diffusion of ions and small clusters leads to Ostwald ripening and hence to discontinuity in the conductive network.

Titanium deposited by FCVA forms a thin layer on the surface composed of small clusters that oxidize within few hours. Concerning the LEI implanted titanium samples a small increase of resistance less than $0.1 \%$ was observed. Figure 4 shows that the dark layer of the implanted titanium ions is located deeper under the surface and is thus better protected from oxidation. Also stiffening (and probable higher density) of the implanted PDMS layer was observed caused by the radiation induced structural changes. This may prevent the diffusion of the oxygen and the diffusion of the implanted ions.

At high doses the electrical properties of all the samples didn't approach thin film properties, but were showing a saturation of conductivity. For FCVA implantations, AFM observations revealed that, despite the high dose, the metal ions on the surface didn't meld into a continuous film, but stayed clustered. In the case of LEI, the sputtering and the cracking of the surface, due to the increased hardness, prevent the formation of a conductive path.

\section{$\underline{\text { Microstructure }}$}

The microstructure of the nanocomposites is shown in figure 3. Chemical and structural modifications of polymers, and therefore the change of the physical properties, as a function of energy, dose or element have already been studied by other groups [4-8]. The energy transferred from an incoming ion to the polymer matrix can be orders of magnitude higher than typical binding energies of a polymer $(15 \mathrm{eV})$. The bond breaking, producing free radicals, excited 
species and volatiles, is caused by excitation and ionization of the polymer molecules as well as direct nuclear collisions. If there is a high concentration of primary radicals, then scissioned polymeric bonds react with each other, recombining or initiating cross-linking. Ti implantation with LEI heavily damaged the implanted layer. This leads to cracks in the implanted membrane, which are due to the increased hardness of PDMS. Starting with the surface roughness of $2 \mathrm{~nm}$ for a virgin membrane, once implanted the surface buckles into an intricate wavy pattern with root mean square height values of several hundred nanometers (see figure 5).

LEI prepared samples fit quite well with distributions presented in figure 1. FCVA images show that the "implantation" ions are also deposited on the surface, because of the drop of the accelerating potential during each pulse. One also may observe that titanium ions are homogeneously distributed in the matrix forming an amorphous composite, whereas gold clusters into crystalline $30 \mathrm{~nm}$ round particles, whose size increases with dose and energy. The reason for this lies in the high chemical reactivity of titanium and the high stability of gold.

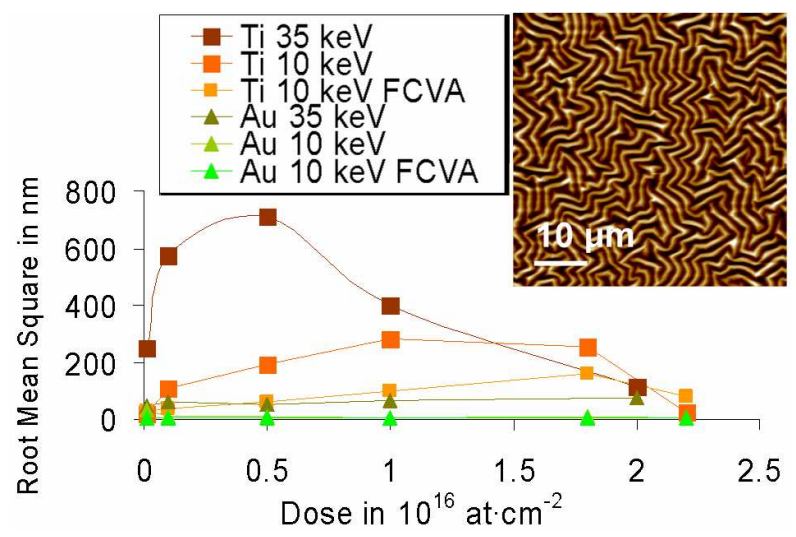

Figure 5. Surface roughness vs. ion dose. Inset: AFM topography for $10 \mathrm{keV}$ FCVA implantation of Au with $2.3 \times 10^{16}$ at $/ \mathrm{cm}^{2}$. The PDMS has initial surface roughness of $2 \mathrm{~nm}$. Post irradiation the roughness is due to the different coefficient of thermal expansion between the implanted and non-implanted layer of PDMS. Owing to the very high concentration of ions on the surface, building a continuous metallic layer (see TEM), the roughness decreases again at the higher doses.

\section{$\underline{\text { Elasticity }}$}

The percolation model, described above, elucidates also the changing of elasticity. At the percolation threshold the Young's modulus increases suddenly as a result of the interconnecting metallic clusters (see titanium in figure 6). Nevertheless for a given dose, important differences are observed between LEI and FCVA, as well as between gold and titanium. The low values of the Young's modulus measured on FCVA samples are explained by a partial deposition of the ions thanks to the energy spread from $50 \mathrm{eV}$ to $10 \mathrm{keV}$. LEI, on the other hand, implants with a mono-energetic beam at $10 \mathrm{keV}$ or $35 \mathrm{keV}$, rastering and heating the surface of PDMS for several hours. This is a big difference from the short pulsed implantation of a few minutes with FCVA; the influence of the energy on the chemical structure has been shortly summarized above. The Young's modulus for Au rises linearly with dose, contrarily to Ti, whose behavior can be explained with the percolation theory. This is probably due to the diffusion of gold. 

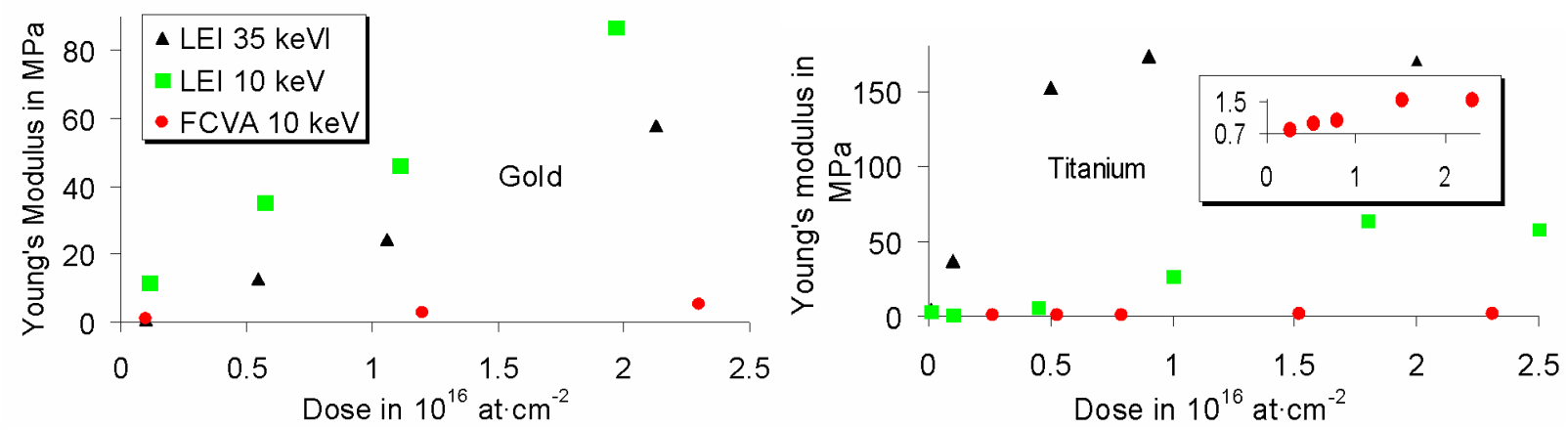

Figure 6. Young's modulus of PDMS membranes after the implantation with Au and Ti. Membranes thickness is $25 \mu \mathrm{m}$ and the initial value of the Young's modulus is $0.85 \mathrm{MPa}$. Metal ion implantation has two main effects: formation of metallic clusters in the PDMS, and irradiation-induced chemical modification of the PDMS. A clear percolation threshold is seen for titanium, but not for gold.

\section{CONCLUSIONS}

FCVA implantation of gold is best suited for creating conductive electrodes in elastomers since it allows the lowest resistivity $\left(300 \Omega\right.$ /square) to be reached at the smallest dose $\left(1.75 \times 10^{16}\right.$ $\mathrm{at} / \mathrm{cm}^{2}$ ) and hence with only limited stiffening of the membrane ( $\left.5 \mathrm{MPa}\right)$. This presents the best combination of high electrical conductivity and low compliance that are the main parameters for artificial muscle actuators and flexible electronics.

\section{ACKNOWLEDGMENTS}

We thank Dr. M. Doebeli (PSI, Ion Beam Physics) for the RBS measurements and Mr. I. Winkler for his work with LEI at the Center for Application of Ion Beams in Materials Research, Forschungszentrum Dresden-Rossendorf (FZR), Germany. This project was funded by the Swiss National Science Foundation grant \#20020-120164, and by the EPFL.

\section{REFERENCES}

1. S. Rosset, M. Niklaus, P. Dubois, Herbert R. Shea, Proceedings of SPIE 7287 (2009).

2. M. Niklaus, S. Rosset, P. Dubois, Herbert R. Shea, Scripta Materialia 59, 893-896 (2008).

3. J. F. Ziegler, The Stopping \& range of ions in matter (2008). URL www.srim.org.

4. H. Dong, T. Bell, Surface and Coatings Technology 111, 29-40 (1999).

5. W. Yuguang, Z. Tonghe, L. Andong, Z. Gu, Surface and Coat. Techn. 157, 262-266 (2002).

6. W. Yuguang, Z. Tonghe, L. Andong, Z. Xu, Z. Gu, Vacuum 69, 461-466 (2003).

7. W. Yuguang, Z. Tonghe, Z.Gu, Z. Huixing, Z.Xiaoji, Surf.\&Coat.Tech. 148, 221-225 (2001).

8. P. K. Chu, J. Y. Chen, L. P. Wang, N. Huang, Materials Science \& Engeneering, Reports 36, 143-206, (2002). 